\title{
Matriptase and survivin expression associated with tumor progression and malignant potential in breast cancer of Chinese women: Tissue microarray analysis of immunostaining scores with clinicopathological parameters
}

\author{
Wen-Chiuan Tsai ${ }^{\mathrm{a}}$, Chi-Hong Chu ${ }^{\mathrm{b}}$, Cheng-Pin Yu ${ }^{\mathrm{a}}$, Lai-Fa Sheu ${ }^{\mathrm{a}}$, Ann-Chen ${ }^{\mathrm{a}}$, Hung Chiang $^{\mathrm{c}}$ and \\ Jong-Shiaw Jin ${ }^{\mathrm{a}, *}$ \\ ${ }^{a}$ Department of Pathology, Tri-Service General Hospital, National Defense Medical Center, Taipei, Taiwan \\ ${ }^{\mathrm{b}}$ Division of General Surgery, Department of Surgery, Tri-Service General Hospital, National Defense Medical \\ Center, Taipei, Taiwan \\ ${ }^{\mathrm{c}}$ Taipei Institute of Pathology, Tri-Service General Hospital, National Defense Medical Center, Taipei, Taiwan
}

\begin{abstract}
Objective: The aim of this study was to examine the expression of matriptase and survivin in breast carcinoma and correlate with clinicopathological parameters.

Methods: Immunohistochemical analysis of matriptase and survivin were performed in tissue microarray slides of 290 cases, including 11 normal breast tissue; 27 fibrocystic disease; 17 fibroadenoma; 6 atypical ductal hyperplasia; 39 ductal carcinoma in situ, low grade (DCIS, low grade); 39 ductal carcinoma in situ, high grade (DCIS, high grade); 27 invasive ductal carcinoma, grade I (IDC, grade I); 78 invasive ductal carcinoma, grade II (IDC, grade II); and 46 invasive ductal carcinoma, grade III (IDC, grade III).

Results: The average immunostaining scores of matriptase were 44.1 in normal breast tissue, 52.7 in fibrocystic disease, 76.5 in fibroadenoma, 81.7 in atypical ductal hyperplasia, 133.7 in low-grade DCIS, and 155.8 in high-grade DCIS. Among 151 breast IDC cases, the average immunostaining scores of matriptase were 172.7 in grade I, 211.7 in grade II, and 221.2 in grade III. Additionally, the average immunostaining scores of surviving also correlate with tumor grades and stages.

Conclusions: Higher expressions of matriptase and survivin correlate significantly with clinicopathological parameters in breast cancer and the malignant potential in premalignant lesions. In addition, higher survivin expression had poorer prognosis of breast IDC cases.
\end{abstract}

Keywords: Atypical ductal hyperplasia, ductal carcinoma in situ, invasive ductal carcinoma, matriptase, survivin

\footnotetext{
* Corresponding author: Jong-Shiaw Jin, M.D., Ph.D., Department of Pathology, Tri-Service General Hospital, National Defense Medical Center, No. 325, Sec. 2, Cheng-Kung Road, Neihu 114, Taipei, Taiwan. Tel.: +886 2 87927155; Fax: +886 2 26913324; E-mail: jsjin@ndmctsgh.edu.tw.
}

\section{Introduction}

Invasive breast cancer is one the most common carcinomas in women, especially in the West. Etiologies of breast cancer include family history, diet, and hormonal imbalance [1]. Multiple factors, such as tumor histopathological grades and subtypes, clinical stages, and lymph node metastasis have been proved to play 
important roles in predicting prognosis and determining adjuvant treatment [1]. New prognostic factors include distinct breast cancer subgroups with different biological features [2]. Premalignant breast disease, such as atypical ductal hyperplasia, is an important predictor of later malignancy [3]. Several immunohistochemical markers, such as p53, Her2/neu, EGFR, estrogen receptor, E-cadherin, have been evaluated for discrimination of breast intraepithelial proliferative lesions, but only cytological evidence has been effective [3]. The development of a novel biomarker to prevent subjective error may be necessary to assist pathologists in making accurate diagnoses.

Matriptase is a multi-domain serine protease that has been detected in several normal-surface epithelia [4-6]. Matriptase was first isolated as a type II transmembrane serine protease expressed in normal breast tissue and carcinoma cells [7-9]. Activation of hepatocyte growth factor (HGF), urokinase plasminogen activator (uPA), and PAR- 2 by matriptase cleaves the extracellular matrix and can induce tumor invasiveness and metastasis [10-12]. Higher expression of matriptase has been proved to be associated with tumor progression in uterine cervical and ovarian cancer [13,14].

Survivin is a $16.3 \mathrm{kD}$ protein consisting of 142 amino acids and belongs to the protein family that inhibits cellular apoptosis [17]. Abundant embryonic tissue and most malignant cells significantly increase levels of survivin expression [18]. Survivin has multifunctional effects on cell proliferation [19], angiogenesis [20-22] and inhibition of apoptosis [18], but detailed mechanisms are still vague [23]. In a recent study, expression of survivin was revealed to be associated with Her2/neu, vascular endothelial growth factor (VEGF), uPA and PAI-1 elevation [23]. Although several cellculture studies have been performed, correlation between survivin and the clinicopathological parameters of breast cancer are still paradoxical [23-27].

In this study, we tested the hypothesis that higher expression of matriptase and survivin have an effect on prognostic significance in breast carcinoma and malignant potential in breast hyperplastic lesions. In addition, we compared the expression of these two biomarkers in breast neoplasms to investigate whether or not co-upregulation effects exist.

\section{Materials and methods}

Paraffin-embedded tumor tissues were obtained from the Department of Pathology, Tri-Service General Hos- pital, and tissue microarray slides were constructed. Two hundred and ninety cases were involved in this study, including 11 cases with normal breast tissue, 27 with fibrocystic disease, 17 fibroadenoma, 6 atypical ductal hyperplasia, 39 low-grade DCIS, 39 high-grade DCIS, 27 grade I IDC, 78 grade II IDC, and 46 grade III IDC. Histopathological gradings of ductal carcinoma in situ and invasive carcinoma were determined according to criteria of the WHO grading system [1].

One core was taken from a selected area of each paraffin-embedded tumor tissue and tissue microarray slides were constructed. Each representative core in the tissue microarray slide was $2 \mathrm{~mm}$ in diameter and the pathological diagnosis in these cases was reviewed by at least two experienced pathologists. Tissue microarray slides showed uniform staining representative of the original paraffin-embedded specimens. All tumors were pathologically staged according to the 1997 American Joint Committee on Cancer (AJCC/TNM system). None of these cases had ever received radiation or chemotherapy before surgery.

\subsection{Immunohistochemistry}

Tissue microarray sections were de-waxed in xylene, rehydrated in alcohol, and immersed in 3\% hydrogen peroxide for 5 minutes to suppress endogenous peroxidase activity. Antigen retrieval was performed by heating $\left(100^{\circ} \mathrm{C}\right)$ each section for 30 minutes in $0.01 \mathrm{~mol} / \mathrm{L}$ sodium citrate buffer ( $\mathrm{pH}$ 6.0). After 3 rinses (each for 5 minutes in phosphate buffered saline [PBS]), sections were incubated for 1 hour at room temperature with a rabbit anti-human matriptase/ST14 antibody (1:100, BETHYL Laboratories, Montgomery, TX, USA) and a rabbit anti-human survivin antibody (1:200, R\&D System, Wiesbaden, Germany) diluted in PBS. After 3 washes (each for 5 minutes in PBS), sections were incubated with biotin-labeled secondary immunoglobulin (1:100, DAKO, Glostrup, Denmark) for 1 hour at room temperature. After 3 additional washes, peroxidase activity was developed with AEC + substrate chromogen (DAKO, Glostrup, Denmark) at room temperature. Sections of normal stromal tissue of breast were used as negative controls.

For evaluation of immunoreactivity and histological appearance, all tissue microarray experiments were repeated two times and the slides were examined and scored by two authors concurrently. For assessment of matriptase and survivin immunostaining scores, the intensity of cytoplasmic and membranous immunostaining was scored on a scale of 0 (no staining) to 4 
Table 1

Matriptase and survivin immunostaining scores in breast disease

\begin{tabular}{lllc}
\hline & \% staining & Intensity & Total score \\
\hline Matriptase expression & & & \\
FCD $(n=27)$ & $43.8 \pm 5.9$ & $1.0 \pm 0.1$ & $52.7 \pm 7.7$ \\
Fibroadenoma $(n=17)$ & $66.5 \pm 6.6$ & $1.1 \pm 0.1$ & $76.5 \pm 10.9$ \\
ADH $(n=6)$ & $66.8 \pm 10.7$ & $1.2 \pm 0.2$ & $81.7 \pm 13.4^{*}$ \\
DCIS, low grade $(n=39)$ & $80.8 \pm 4.9^{*}$ & $1.6 \pm 0.1^{*}$ & $133.7 \pm 14.7^{*+}$ \\
DCIS, high grade $(n=39)$ & $81.1 \pm 3.5^{*}$ & $2.0 \pm 0.2^{*}$ & $155.8 \pm 15.7^{*+}$ \\
IDC, grade I $(n=27)$ & $82.3 \pm 4.6^{*}$ & $2.1 \pm 0.2^{*}$ & $172.7 \pm 14.8^{*+}$ \\
IDC, grade II $(n=78)$ & $87.4 \pm 1.9^{*}$ & $2.4 \pm 0.1^{*}$ & $211.7 \pm 11.3^{*+}$ \\
IDC, grade III $(n=46)$ & $90.7 \pm 1.8^{*}$ & $2.4 \pm 0.2^{*}$ & $221.2 \pm 16.2^{*+}$ \\
Normal breast tissue $(n=11)$ & $35.9 \pm 9.9$ & $0.9 \pm 0.2$ & $44.1 \pm 15.4$ \\
Survivin expression & & & \\
FCD $(n=27)$ & $69.4 \pm 4.0$ & $1.6 \pm 0.1$ & $113.1 \pm 8.6^{*}$ \\
Fibroadenoma $(n=17)$ & $75.6 \pm 5.2$ & $1.9 \pm 0.2$ & $150.6 \pm 19.2^{*}$ \\
ADH $(n=6)$ & $76.7 \pm 7.6$ & $2.0 \pm 0.3$ & $161.7 \pm 19.9^{*}$ \\
DCIS, low grade $(n=39)$ & $85.7 \pm 2.8^{*}$ & $2.3 \pm 0.1^{*}$ & $198.4 \pm 12.0^{*+}$ \\
DCIS, high grade $(n=39)$ & $86.4 \pm 2.6^{*}$ & $2.5 \pm 0.2^{*}$ & $217.8 \pm 18.4^{*+}$ \\
IDC, grade I $(n=27)$ & $87.3 \pm 3.4^{*}$ & $2.7 \pm 0.2^{*}$ & $240.7 \pm 21.0^{*+}$ \\
IDC, grade II $(n=78)$ & $88.6 \pm 1.7^{*}$ & $2.8 \pm 0.1$ & $247.7 \pm 12.5^{*+}$ \\
IDC, grade III $(n=46)$ & $90.4 \pm 1.9^{*}$ & $3.0 \pm 0.4^{*}$ & $268.0 \pm 13.7^{*+}$ \\
Normal breast tissue $(n=11)$ & $55.5 \pm 6.2$ & $1.3 \pm 0.2$ & $64.5 \pm 4.7$ \\
\hline Dat aremean $(n+5 n a t$
\end{tabular}

Data are means \pm standard error of the mean (SEM) of the matriptase and survivin immunostaining scores in normal breast tissue and neoplasm. ${ }^{*} p<0.05$ vs. normal breast tissue. ${ }^{+} p<0.05$ vs. atypical ductal hyperplasia.

Abbreviations: fibrocystic disease, FCD; atypical ductal hyperplasia, ADH; ductal carcinoma in situ, DCIS; invasive duct carcinoma, IDC.

(strongest intensity), and the percentage of cells with stained cytoplasm or plasma membrane was estimated at each intensity. The percentage of cells (from 0 to 100) was multiplied by the corresponding immunostaining intensity (from 0 to 4 ) to obtain an immunostaining score ranging from 0 to 400 .

\subsection{Statistical analysis}

All results are expressed as mean \pm standard error of the mean (S.E.M.). The immunostaining scores of matriptase and survivin in breast diseases were compared with the score in normal breast epithelia. Statistical analysis was performed using the ANOVA test between groups. A p-value of less than 0.05 was considered to be statistically significant. SigmaState software (Jandel Scientific, San Rafael, CA, USA) was used to perform linear regression testing and Pearson or Sperman correlation test to analyze the relationship between expression of these two biomarkers and clinicopathological parameters in breast IDC cases.

In addition, survival time of subjects was calculated from the date of surgery to the date of death. 121 of 151 IDC patients in the study received 5-year follow up; subjects were divided into 2 groups in order to compare survival times with matriptase and survivin immunostaining scores. Statistical analysis of survival time was done using the Kaplan-Meier survival test.

\section{Results}

\subsection{Clinicopathological characteristics}

Among all 151 breast IDC cases, age distribution ranged from 27 to 89 years and mean age was 54.3. The average tumor size was $3.5 \mathrm{~cm}$ in diameter. Other information, including histopathological grading and staging distribution, are shown in Tables 1, 2 and 3.

\subsection{Matriptase expression in breast diseases}

Matriptase immunoreactivity was seen on the cell membrane and cytoplasm of all 290 cases, with varying intensities and percentages (Table 1). The average matriptase immunostaining score was 44.1 in normal breast epithelium (Fig. 1A-1B); 52.7 in fibrocystic disease (Fig. 1D-1E); 76.5 in fibroadenoma (Fig. 1G-1H); 81.7 in atypical ductal hyperplasia (Fig. $1 \mathrm{~J}-1 \mathrm{~K}) ; 133.7$ in DCIS, low grade (Fig. 1M-1N); and 155.8 in DCIS, high grade (Fig. 1P-1Q). In addition, among 151 IDC cases, the average immunostaining scores of matriptase were 172.7 for grade I (Fig. 2A-2B), 211.7 for grade II (Fig. 2D-2E), and 221.2 for grade III (Fig. 2G-2H). Through a series of statistical analyses, significant positive correlation of matriptase staining scores with his- 
Table 2

The immunostaining patterns of matriptase and clinicopatholigcal parameters of breast invasive ductal carcinoma

\begin{tabular}{cccccl}
\hline TNM stage & $\begin{array}{c}\text { No. of } \\
\text { cases }\end{array}$ & $\begin{array}{c}\text { Average } \\
\text { intensity }\end{array}$ & $\begin{array}{c}\text { Average } \\
\% \text { tumor }\end{array}$ & $\begin{array}{c}\text { Average } \\
\text { score }\end{array}$ & Correlation \\
\hline T stage & & & & & \\
T1 & 39 & 2.0 & 80.9 & 168.6 & \\
T2 & 49 & 2.2 & 88.4 & 196.8 & Positive correlation $(P=0.009)$ \\
T3 & 37 & 2.5 & 88.7 & 223.1 & \\
T4 & 26 & 2.7 & 89.0 & 244.5 & \\
N stage & & & & & \\
N0 & 47 & 2.0 & 83.1 & 171.9 & \\
N1 & 46 & 2.2 & 86.7 & 194.6 & Positive correlation $(P=0.011)$ \\
N2 & 32 & 2.6 & 87.3 & 232.1 & \\
N3 & 26 & 2.6 & 91.7 & 245.6 & \\
M stage & & & & & \\
M0 & 107 & 2.2 & 85.4 & 198.0 & No correlation $(P=0.30)$ \\
M1 & 44 & 2.4 & 86.3 & 218.0 & \\
AJCC stage & & & & & \\
Stage I & 36 & 1.9 & 81.1 & 161.1 & \\
Stage II & 49 & 2.0 & 87.1 & 176.3 & Positive correlation $(P=0.038)$ \\
Stage III & 22 & 2.4 & 89.3 & 215.7 & \\
Stage IV & 44 & 2.4 & 89.5 & 218.0 & \\
\hline
\end{tabular}

Table 3

The immunostaining patterns of survivin and clinicopatholigcal parameters of breast invasive ductal carcinoma

\begin{tabular}{|c|c|c|c|c|c|}
\hline TNM stage & $\begin{array}{c}\text { No. of } \\
\text { cases }\end{array}$ & $\begin{array}{l}\text { Average } \\
\text { intensity }\end{array}$ & $\begin{array}{l}\text { Average } \\
\% \text { tumor }\end{array}$ & $\begin{array}{c}\text { Average } \\
\text { score }\end{array}$ & Correlation \\
\hline \multicolumn{6}{|l|}{ T stage } \\
\hline $\mathrm{T} 1$ & 39 & 1.6 & 82.0 & 132.2 & \multirow{4}{*}{ Positive correlation $(P=0.005)$} \\
\hline $\mathrm{T} 2$ & 49 & 2.0 & 86.9 & 175.8 & \\
\hline $\mathrm{T} 3$ & 37 & 2.4 & 93.3 & 224.0 & \\
\hline $\mathrm{T} 4$ & 26 & 3.3 & 95.2 & 317.5 & \\
\hline \multicolumn{6}{|l|}{$\mathrm{N}$ stage } \\
\hline No & 47 & 1.8 & 84.4 & 150.8 & \multirow{4}{*}{ Positive correlation $(P=0.028)$} \\
\hline N1 & 46 & 2.0 & 87.4 & 173.7 & \\
\hline $\mathrm{N} 2$ & 32 & 2.7 & 91.4 & 245.9 & \\
\hline N3 & 26 & 3.3 & 93.5 & 298.8 & \\
\hline \multicolumn{6}{|l|}{ M stage } \\
\hline M0 & 107 & 2.8 & 87.0 & 210.4 & \multirow{2}{*}{ Positive correlation $(P=0.047)$} \\
\hline M1 & 44 & 3.1 & 94.8 & 295.6 & \\
\hline \multicolumn{6}{|l|}{ AJCC stage } \\
\hline Stage I & 36 & 1.6 & 82.4 & 133.0 & \multirow{4}{*}{ Positive correlation $(P=0.017)$} \\
\hline Stage II & 49 & 2.0 & 88.3 & 172.0 & \\
\hline Stage III & 22 & 2.7 & 92.4 & 248.6 & \\
\hline Stage IV & 44 & 3.1 & 94.8 & 295.6 & \\
\hline
\end{tabular}

tological grading system of breast IDC was confirmed (Table $1, P<0.05$ )

Among all breast IDC cases, 39 cases of stage T1 were found, 49 stage $\mathrm{T} 2$ cases, 37 stage T3 cases, and 26 stage $\mathrm{T} 4$ cases. In this study, more advanced stages of breast IDC had higher matriptase intensity and immunostaining scores. The average immunostaining score of matriptase was 168.6 in stage $\mathrm{T} 1,196.8$ in stage T2, 223.1 in stage T3, and 244.5 in stage T4 cases of breast IDC. Positive correlation of matriptase staining scores with $\mathrm{T}$ stages was validated based on $\mathrm{P}$ values of less than 0.05 . In addition, more advanced
$\mathrm{N}$ (nodal) stages of breast IDC are also associated with higher intensity, greater percentage of tumor staining and immunostaining scores of matriptase expression. Finally, we divided breast IDC cases from stages I to IV based on the clinical staging system. The immunostaining scores of various clinical stages of breast IDC are as follows: 161.1 for stage I, 176.2 for stage II, 215.7 for stage III, and 218.0 for stage IV. The immunostaining scores of matriptase positively correlated with the clinical staging system $(P<0.05$, Table 2 and Fig. 3). 

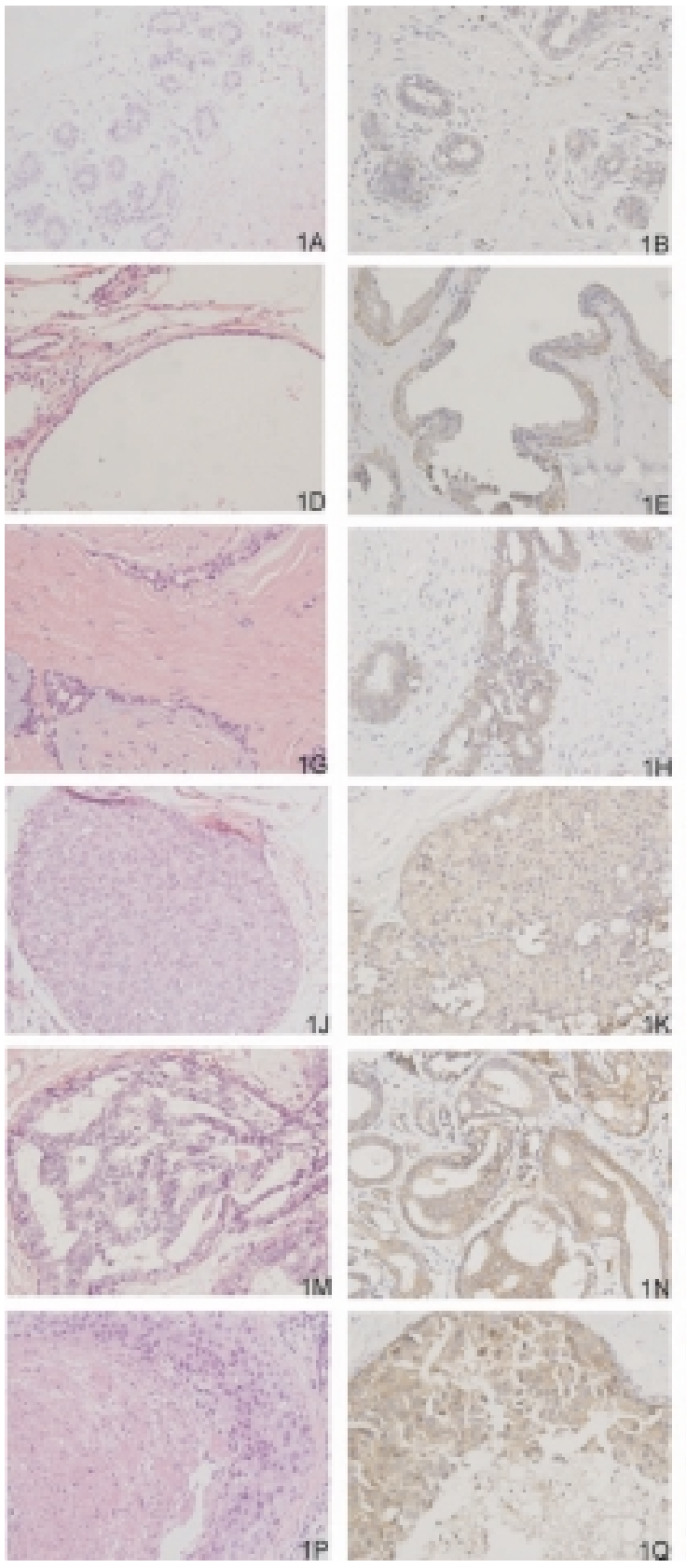
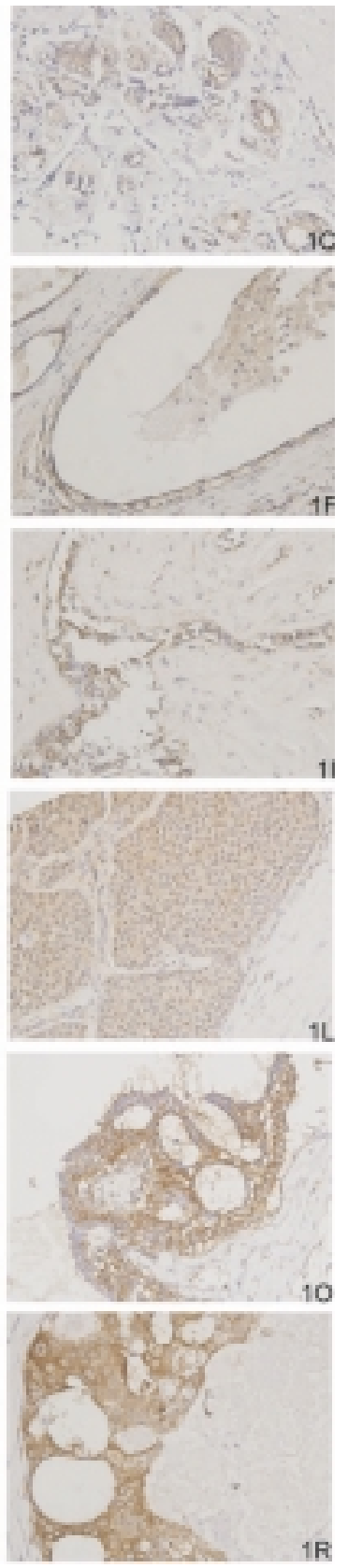

Fig. 1. Hematoxylin and eosin staining of normal breast tissue (1A), fibrocystic disease (1D), fibroadenoma (1G), atypical ductal hyperplasia (1J), low-grade DCIS (1M), and high-grade DCIS (1P). Immunohistochemical analysis of matriptase in normal breast tissue (1B), fibrocystic disease (1E), fibroadenoma $(1 \mathrm{H})$, atypical ductal hyperplasia $(1 \mathrm{~K})$, low-grade DCIS (1N), and high-grade DCIS (1Q). Immunoexpression of survivin in normal breast tissue (1C), fibrocystic disease (1F), fibroadenoma (1I), atypical ductal hyperplasia (1L), low-grade DCIS (1O), and high-grade DCIS (1R). (Original magnification X 400).

\subsection{Survivin expression in breast diseases}

The immunostaining scores of survivin in normal breast tissue and various diseases are shown in Tables 1 and 3. Survivin immunoreactivities were present on the cell membrane and cytoplasm of all 290 cases, with varying intensities and percentages of tumor cells. Immunostaining scores of survivin were 64.5 in normal mammary glands (Fig. 1C), 113.1 in fibrocystic disease (Fig. 1F), 150.6 in fibroadenoma (Fig. 1I), 

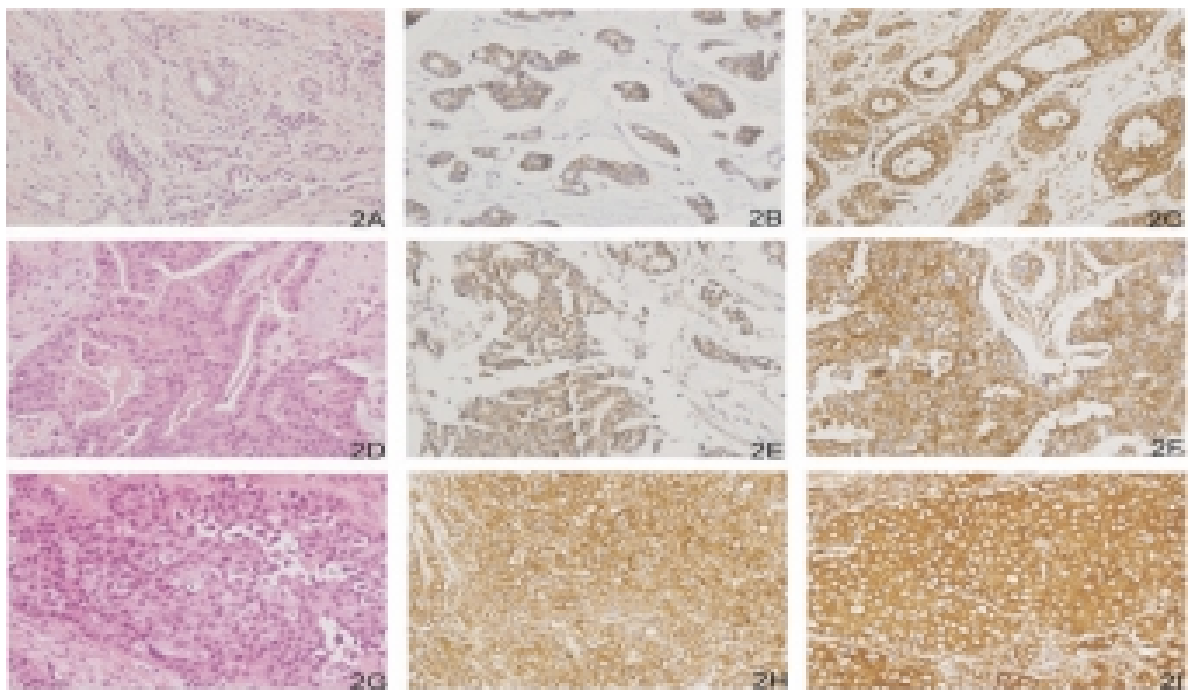

Fig. 2. Hematoxylin and eosin staining of IDC, grade I (2A); IDC, grade II (2D); and IDC, grade III (2G). Immunohistochemical analysis of matriptase in IDC, grade I (2B), IDC, grade II (2E), and IDC, grade III (2H). Immunoexpression of survivin in IDC, grade I (2C), IDC, grade II (2F), and IDC, grade III (2I). (Original magnification X 400).
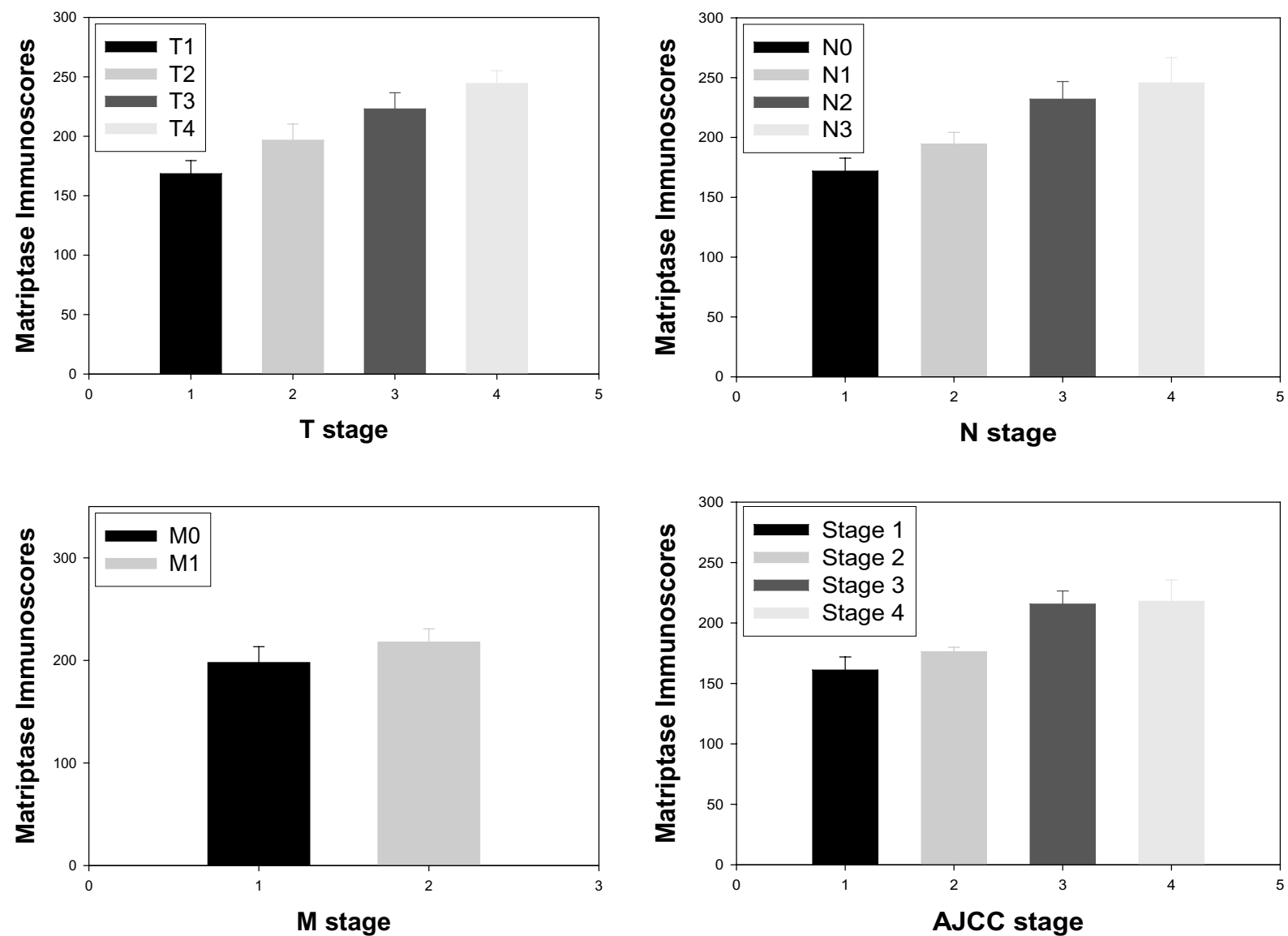

Fig. 3. Clinicopathological correlations with matriptase immunostaining scores in breast IDC. 

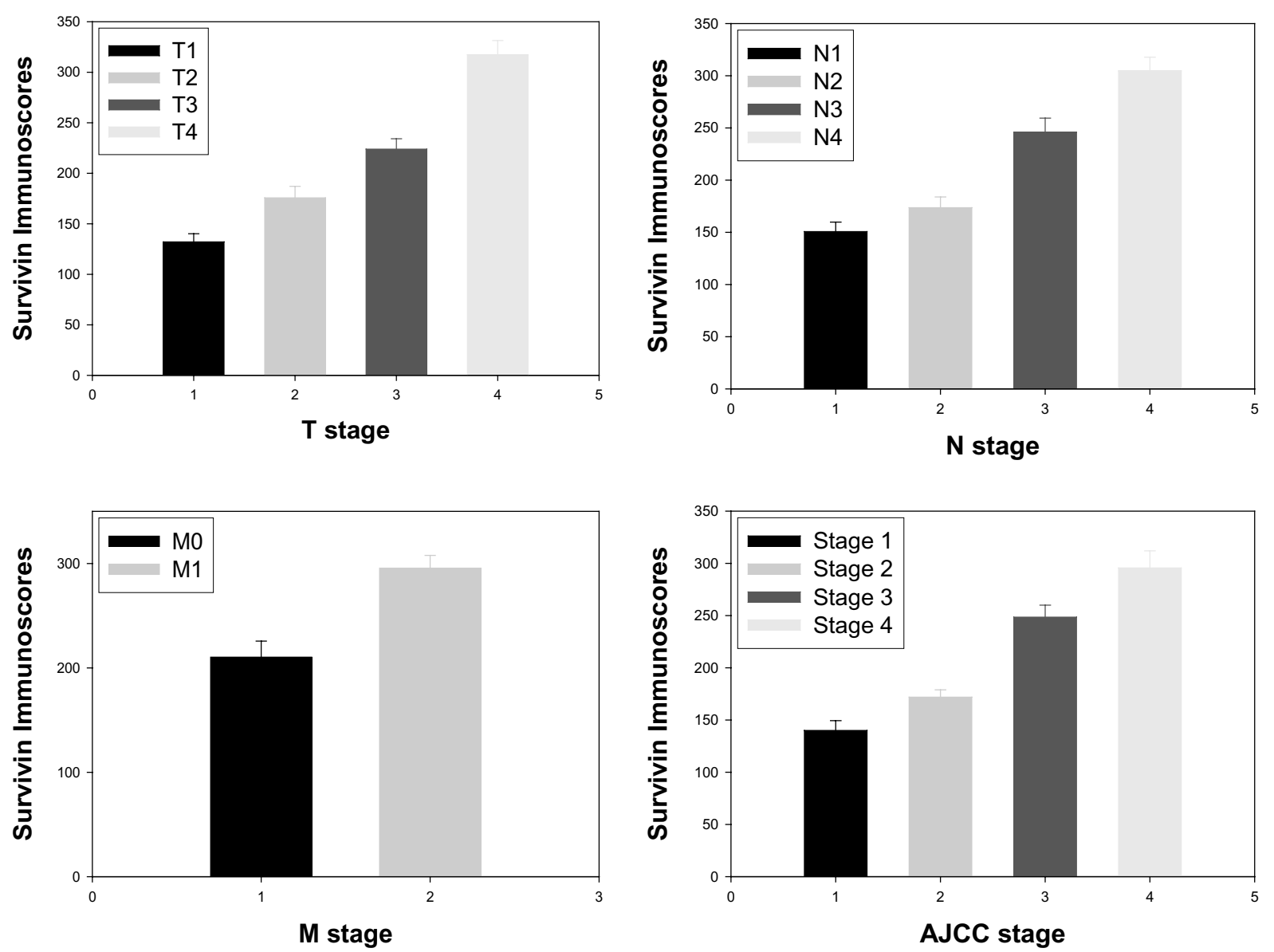

Fig. 4. Clinicopathological correlations with survivin immunostaining scores in breast IDC.

161.7 in atypical ductal hyperplasia (Fig. 1L), 198.4 in low-grade DCIS (Fig. 1O), and 217.8 in high-grade DCIS (Fig. 1R). Among 151 breast cancer cases, average immunostaining scores were 240.7 in IDC, grade I (Fig. 2C); 247.7 in IDC, grade II (Fig. 2F); and 268.0 in IDC, grade III (Fig. 2I). Statistical analysis showed positive correlation of survivin immunostaining scores with histological grading of breast cancer (Table $1, P<$ 0.05).

Average immunostaining scores of survivin were 132.2 for stage T1, 175.8 for stage T2, 224.0 for stage $\mathrm{T} 3$, and 317.5 for stage T4 cases of breast IDC. T (tumor size) stages correlated significantly with higher survivin immunostaining scores $(P<0.05)$. In addition, more advanced $\mathrm{N}$ and $\mathrm{M}$ stages of breast IDC cases had significantly higher immunostaining scores of survivin expression. Immunostaining scores of survivin in various clinical stages of breast cancer cases are as follows: 133.0 for stage I, 172.0 for stage II, 248.6 for stage III, and 295.6 for stage IV. Immunos- taining scores of survivin also correlated significantly with the clinical staging system $(P<0.05$, Table 3 and Fig. 4).

\subsection{Relationship of matriptase and survivin expression with survival time}

We divided 121 RCC cases that had received 5-year follow up into two groups based on matriptase and survivin immunoscores, respectively. According to matriptase immunoscores, 65 cases had higher expression (immunostaining score $\geqslant 210$ ) in one group and, in the other group, the remaining 56 cases had lower immunoreactivity (immunostaining score $<210$ ). Similarly, these included cases after follow up were also divided into two groups based on survivin immunostaining scores. There were 58 cases of higher survivin expression (immunostaining score $\geqslant 250$ ) and 63 cases of lower survivin immunoactivity (immunostaining score $<250$ ). Using matriptase and survivin immunoscores 


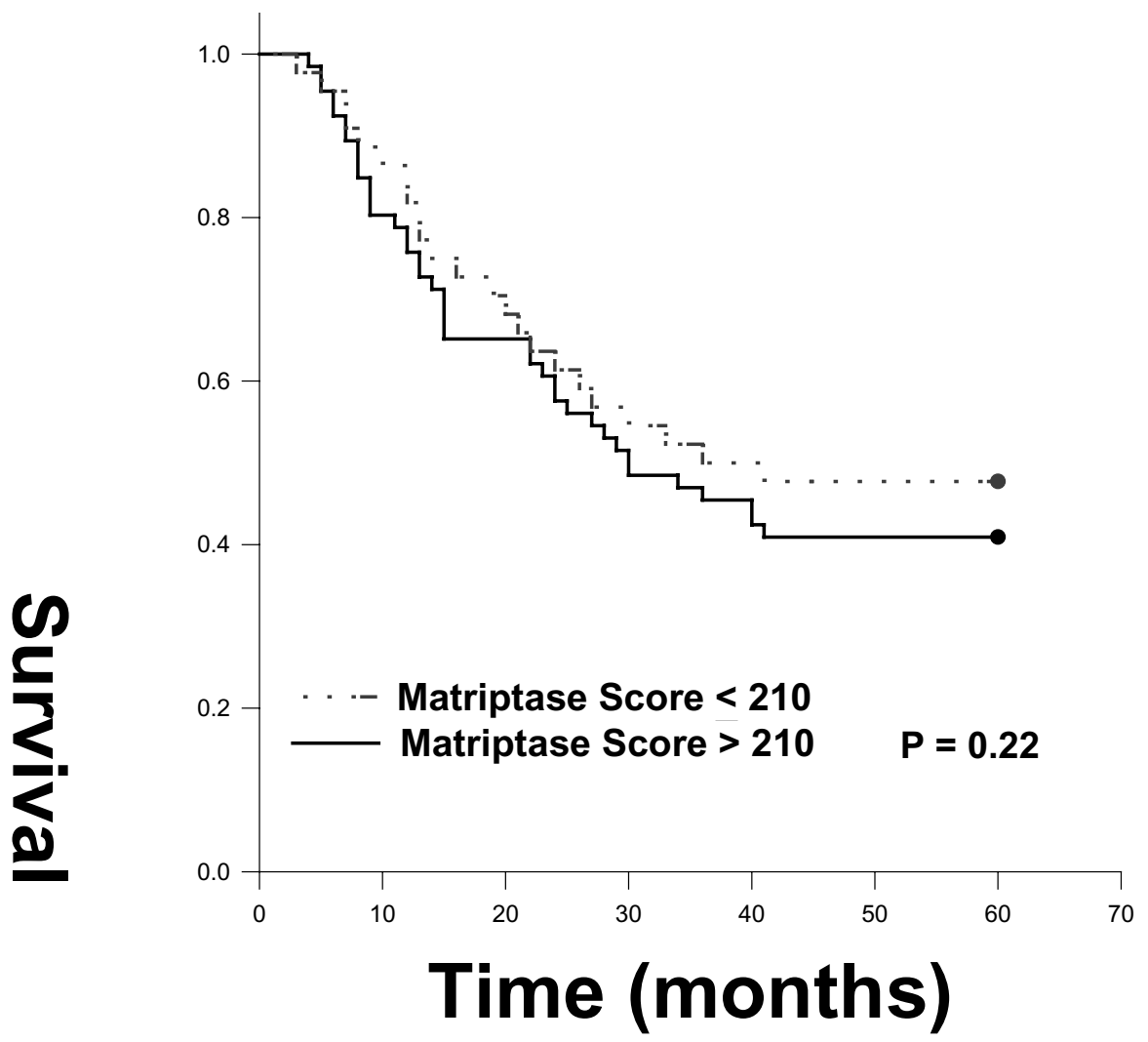

Fig. 5. Overall survival of 121 patients with breast IDC. Higher matriptase immunostaining scores were associated with poorer survival, but did not reach statistical significance. Survival rates were analyzed using the Kaplan-Meier survival test.

as variable parameters, higher scores in these biomarkers were associated with higher mortality, and survivin immunoscores reached statistical significance (Figs 5 and 6).

\subsection{Correlation between matriptase and survivin in breast IDC}

Significantly higher matriptase immunostaining scores correlated positively with survivin immunostaining scores in breast IDC cases. Correlation between matriptase and survivin immunostaining scores for all 151 breast IDC cases is shown in Fig. 7.

\section{Discussion}

Breast cancer is one of the most common cancers, representing more than $25 \%$ of all malignancies in Chinese women [28]. In clinical follow up study, intraductal proliferative lesions of breast may be associated with cancer development. Risks of breast IDC development were 1.5-fold in usual ductal hyperplasia, 4- to 5-fold in atypical ductal hyperplasia, and 8to 10 -fold in DCIS [30]. However, many similarities are still found in ductal hyperplasia and DCIS based on histopathologic features. In several studies, erbB-2 has been proved to be associated with high histological grades of breast cancer, but was shown to have little effect on the distinction between these premalignant lesions [30].

Matriptase is an epithelial-derived, integral serine protease that has been associated with cancer cell invasion and metastasis [31]. Expression of matriptase had been detected in normal human tissue, especially in glandular and squamous epithelia [32]. Latent uPA and pro-HGF are substrates for matriptase. These two substrates both participate in tumor progression through reactions with the extracellular matrix $[10,11]$. Matriptase is highly expressed by various carcinomas, such as cancer of the breast, ovary, colon, endometrium, prostate and stomach $[14,35,36]$. However, even though we see a relationship between matriptase expression and clinicopathological parameters in breast 


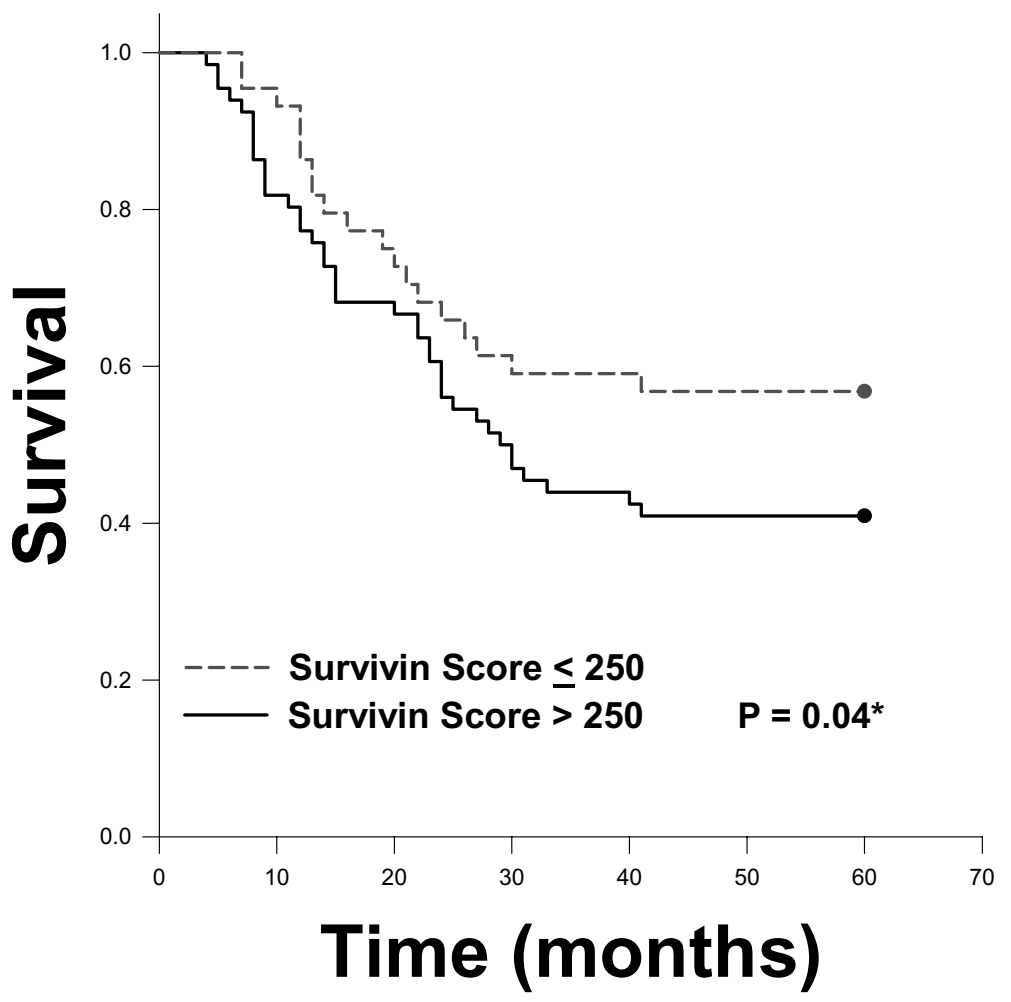

Fig. 6. Overall survival of 121 patients with breast IDC. Higher survivin immunostaining scores were associated with poorer survival, and reach statistical significance. Survival rates were analyzed using the Kaplan-Meier survival test.

cancer, discrimination of intraepithelial proliferative lesions is still vague.

Survivin, an anti-apoptotic factor, is associated with increased tumor aggressiveness and a poorer prognosis in several malignancies, including nasopharyngeal, esophageal, liver, pancreatic, colorectal, renal, urinary bladder, and hematological malignancies [37-44]. In some in vivo studies, survivin expression has been proved to be a prognostic factor $[23,24]$, and the upregulation of survivin may be associated with $\mathrm{p} 53$ gene expression [26]. However, the detailed mechanism of survivin expression is not clear in breast cancer. Direct evidence still lacks to show correlation between survivin expression and clinicopathological staging of the breast cancer in large clinical studies.

In the current study, we first demonstrated that matriptase and survivin immunostaining scores indeed correlated with histological grading and clinical staging in Chinese women with breast cancer. The immunostaining scores of atypical ductal hyperplasia in matriptase were $81.7 \pm 13.4$. It represented that the range of immunostaining scores in $95 \%$ atypical ductal hyperplasia cases was from 68 to 94 . The suggestive cut-off value of immunostaining scores of matrip-

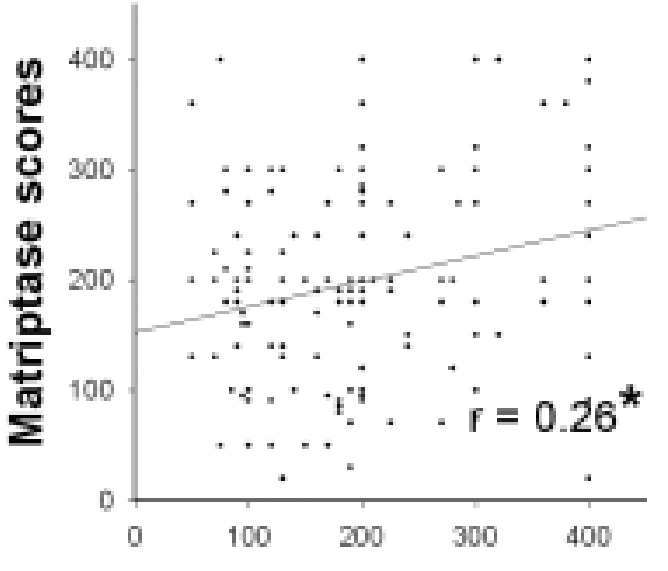

Survivin scores

Fig. 7. Correlation of matriptase immunostaining scores with survivin immunostaining scores in breast IDC cases. * indicates statistical significance of linear regression testing $(p<0.05)$.

tase between atypical ductal hyperplasia and low grade DCIS was 95. Similarly, the suggestive cut-off value of immunostaining scores of survivin between atypical ductal hyperplasia and low grade DCIS was 180 . 
In previous studies, both matriptase and survivin have been proved to be associated with uPA activation, but the relationship between the two was still not clear [14,23]. Our study, on the other hand, demonstrated positive correlation between matriptase and survivin immunohistochemical scores in Chinese women with breast IDC. The up-regulation of matriptase associated with survivin elevation in breast cancer may also have adjuvant effects on tumor progression in breast cancer.

In our study, all tumor tissues were placed in a single tissue array slide. The tissue microarray technique is a powerful tool for simultaneous histological and immunohistochemical evaluation of tumors [45]. Previous studies measuring immunohistochemical intensity of individual cases were limited because of the variability of the chemical signal generated under different environmental conditions [45]. However, the advantage of tissue microarray is that all tissues are under the same conditions and evaluated in one slide [45]. In this study, the clear cut difference seen between epithelial and stromal components in breast tissue by using matriptase or survivin immunostaining revealed the credibility of using tissue microarray slides. Therefore, immunostaining scores used in our study could reflect the relative protein expression levels of matriptase and survivin in breast tissue.

\section{Conclusion}

In conclusion, higher matriptase and survivin immunoscores were correlated significantly with more advanced TNM stages of breast IDC cases. Although there are still unknown mechanisms in tumor progression, we demonstrated that matriptase and survivin were applicable biomarkers to predict clinicopathological parameters. Therefore, the development of pharmacological agents to target the survivin pathway may prolong survival time and slow tumor progression in patients with breast IDC.

\section{Competing interests}

The authors declare that they have no competing interests.

\section{Authors' contribution}

WCT and JSJ designed the study, counted the immunohistochemical scores of these two biomarkers, and reviewed the histopathology in breast cancer. CPY and LFS diagnosed all included cases in this study and graded breast cancer. JCY provided tissue blocks and clinical data. AC performed statistical analysis and HC performed immunohistochemical stains for these cases.

\section{Acknowledgments}

This study was supported by grants from the National Science Counsel, NSC94-2320-B-016-017, and TriService General Hospital, TSGH-C95-16-S05, Taiwan.

\section{References}

[1] F.A. Tavassoli and P. Devilee, eds, World Health Organization Classification of Tumours. Pathology and Genetics of Tumors of the Breast and Female Genital Organs, Lyon: IARC Press, 2004.

[2] N.M. Almasri and M. Al Hamad, Immunohistochemical evaluation of human epidermal growth factor receptor 2 and estrogen and progesterone receptors in breast carcinoma in Jordan, Breast Cancer Res 7 (2005), R598-R604.

[3] J.F. Simpson and D.L. Page, Pathology of preinvasive and excellent prognosis breast cancer, Curr Opin Oncol 13 (2001), 426-430.

[4] C.Y. Lin, J. Anders, M. Johnson et al., Molecular cloning of cDNA for matriptase, a matrix-degrading serine protease with trypsin-like activity, J Biol Chem 274 (1999), 18231-18236.

[5] T. Takeuchi, M.A. Shuman and C.S. Craik, Reverse biochemistry: use of macromolecular protease inhibitors to dissect complex biological processes and identify a membrane-type serine protease in epithelial cancer and normal tissue, Proc Natl Acad Sci USA 96 (1999), 11054-11061.

[6] H. Tanimoto, L.J. Underwood, Y. Wang et al., Ovarian tumor cells express a transmembrane serine protease: a potential candidate for early diagnosis and therapeutic intervention, Tumour Biol 22 (2001), 104-114.

[7] Y.E. Shi, J. Torri, L. Yieh et al., Identification and characterization of a novel matrix-degrading protease from hormonedependent human breast cancer cells, Cancer Res 53 (1993), 1409-1415.

[8] C.Y. Lin, J.K. Wang, J. Torri et al., Characterization of a novel, membrane-bound, 80-kDa matrix-degrading protease from human breast cancer cells. Monoclonal antibody production, isolation, and localization, J Biol Chem 272 (1997), 91479152.

[9] C.Y. Lin, J. Anders, M. Johnson et al., Purification and characterization of a complex containing matriptase and a Kunitztype serine protease inhibitor from human milk, $J$ Biol Chem 274 (1999), 18237-18242.

[10] S.L. Lee, R.B. Dickson and C.Y. Lin, Activation of hepatocyte growth factor and urokinase/plasminogen activator by matriptase, an epithelial membrane serine protease, J Biol Chem 275 (2000), 36720-36725. 
[11] T. Takeuchi, J.L. Harris, W. Huang et al., Cellular localization of membrane-type serine protease 1 and identification of protease-activated receptor- 2 and single-chain urokinase-type plasminogen activator as substrates, J Biol Chem 275 (2000), 26333-26342.

[12] S. Satomi, Y. Yamasaki, S. Tsuzuki et al., A role for membrane-type serine protease (MT-SP1) in intestinal epithelial turnover, Biochem Biophys Res Commun 287 (2001), 9951002 .

[13] J.W. Lee, S. Yong Song, J.J. Choi et al., Increased expression of matriptase is associated with histopathologic grades of cervical neoplasia, Hum Pathol 36 (2005), 626-633.

[14] M.D. Oberst, M.D. Johnson, R.B. Dickson et al., Expression of the serine protease matriptase and its inhibitor HAI-1 in epithelial ovarian cancer: correlation with clinical outcome and tumor clinicopathological parameters, Clin Cancer Res $\mathbf{8}$ (2002), 1101-1107.

[15] C.M. Benaud, M. Oberst, R.B. Dickson et al., Deregulated activation of matriptase in breast cancer cells, Clin Exp Metastasis 19 (2002), 639-649.

[16] C. Parr, G. Watkins, R.E. Mansel et al., The hepatocyte growth factor regulatory factors in human breast cancer, Clin Cancer Res 10 (2004), 202-211.

[17] N.K. Sah, Z. Khan, G.J. Khan et al., Structural, functional and therapeutic biology of survivin, Cancer Lett 244 (2006), $164-171$.

[18] G. Ambrosini, C. Adida and D.C. Altieri, A novel antiapoptosis gene, survivin, expressed in cancer and lymphoma, Nat Med 3 (1997), 917-921.

[19] J.C. Reed and J.R. Bischoff, BIRinging chromosomes through cell division-and survivin' the experience, Cell 102 (2000), 545-548.

[20] J. Tran, J. Rak, C. Sheehan et al., Marked induction of the IAP family antiapoptotic proteins survivin and XIAP by VEGF in vascular endothelial cells, Biochem Biophys Res Commun 264 (1999), 781-788.

[21] O.P. Blanc-Brude, M. Mesri, N.R. Wall et al., Therapeutic targeting of the survivin pathway in cancer: initiation of mitochondrial apoptosis and suppression of tumor-associated angiogenesis, Clin Cancer Res 9 (2003), 2683-2692.

[22] J. Tran, Z. Master, J.L. Yu et al., A role for survivin in chemoresistance of endothelial cells mediated by VEGF, Proc Natl Acad Sci USA 99 (2002), 4349-4354.

[23] B.M. Ryan, G.E. Konecny, S. Kahlert et al., Survivin expression in breast cancer predicts clinical outcome and is associated with HER2, VEGF, urokinase plasminogen activator and PAI-1, Ann Oncol 17 (2006), 597-604.

[24] P.N. Span, F.C. Sweep, E.T. Wiegerinck et al., Survivin is an independent prognostic marker for risk stratification of breast cancer patients, Clin Chem 50 (2004), 1986-1993.

[25] F. Vegran, R. Boidot, C. Oudin et al., Distinct expression of Survivin splice variants in breast carcinomas, Int J Oncol 27 (2005), 1151-1157.

[26] F. Vegran, R. Boidot, C. Oudin et al., Association of p53 gene alterations with the expression of antiapoptotic survivin splice variants in breast cancer, Oncogene (2006), article in press.

[27] P.N. Span, V.C. Tjan-Heijnen, P. Manders et al., High survivin predicts a poor response to endocrine therapy, but a good response to chemotherapy in advanced breast cancer, Breast Cancer Res Treat 98 (2006), 223-230.

[28] M.M. Lee, I.Y. Chang, C.F. Horng et al., Breast cancer and dietary factors in Taiwanese women, Cancer Causes Control 16 (2005), 929-937.
[29] M.D. Pineda, E. White, A.R. Kristal et al., Asian breast cancer survival in the US: a comparison between Asian immigrants, US-born Asian Americans and Caucasians, Int J Epidemiol 30 (2001), 976-982.

[30] P.L. Fitzgibbons, D.L. Page, D. Weaver et al., Prognostic factors in breast cancer. College of American Pathologists Consensus Statement 1999, Arch Pathol Lab Med 124 (2000), 966-978.

[31] M.D. Oberst, L.Y. Chen, K. Kiyomiya et al., HAI-1 regulates activation and expression of matriptase, a membrane-bound serine protease, Am J Physiol Cell Physiol 289 (2005), C462C470.

[32] M.D. Oberst, B. Singh, M. Ozdemirli et al., Characterization of matriptase expression in normal human tissues, J Histochem Cytochem 51 (2003), 1017-1025.

[33] T. Shimomura, K. Denda, A. Kitamura et al., Hepatocyte growth factor activator inhibitor, a novel Kunitz-type serine protease inhibitor, J Biol Chem 272 (1997), 6370-6376.

[34] H. Kataoka, T. Shimomura, T. Kawaguchi et al., Hepatocyte growth factor activator inhibitor type 1 is a specific cell surface binding protein of hepatocyte growth factor activator (HGFA) and regulates HGFA activity in the pericellular microenvironment, J Biol Chem 275 (2000), 40453-40562.

[35] M. Oberst, J. Anders, B. Xie et al., Matriptase and HAI-1 are expressed by normal and malignant epithelial cells in vitro and in vivo, Am J Pathol 158 (2001), 1301-1311.

[36] H. Tanimoto, L.J. Underwood, Y. Wang et al., Ovarian tumor cells express a transmembrane serine protease: a potential candidate for early diagnosis and therapeutic intervention, Tumour Biol 22 (2001), 104-114.

[37] Y. Xiang, H. Yao, S. Wang et al., Prognostic value of Survivin and Livin in nasopharyngeal carcinoma, Laryngoscope 116 (2006), 126-130.

[38] A. Rosato, M. Pivetta, A. Parenti et al., Survivin in esophageal cancer: An accurate prognostic marker for squamous cell carcinoma but not adenocarcinoma, Int J Cancer (2006), article in press.

[39] Zhu H, Chen XP, Zhang WG, et al. Expression and significance of new inhibitor of apoptosis protein survivin in hepatocellular carcinoma. World J Gastroenterol 11 (2005), 3855-3859.

[40] U. Bhanot, R. Heydrich, P. Moller et al., Survivin expression in pancreatic intraepithelial neoplasia (PanIN): steady increase along the developmental stages of pancreatic ductal adenocarcinoma, Am J Surg Pathol 30 (2006), 754-759.

[41] Abd El-Hameed A. Survivin expression in colorectal adenocarcinoma using tissue microarray. J Egypt Natl Canc Inst 2005, 17:42-50.

[42] Mahotka C, Krieg T, Krieg A, et al. Distinct in vivo expression patterns of survivin splice variants in renal cell carcinomas. Int J Cancer 2002, 100:30-6.

[43] Kitamura H, Torigoe T, Honma I, et al. Expression and antigenicity of survivin, an inhibitor of apoptosis family member, in bladder cancer: implications for specific immunotherapy. Urology 2006, 67:955-9.

[44] Invernizzi R, Travaglino E, Benatti C, et al. Survivin expression, apoptosis and proliferation in chronic myelomonocytic leukemia. Eur J Haematol 2006, 76:494-501.

[45] Lam JS, Belldegrum AS, Figlin RA. Tissue array-based predictions of pathobiology, prognosis and response to treatment for renal cell carcinoma therapy. Clin Cancer Res 2004, 10:6304s-6309s 


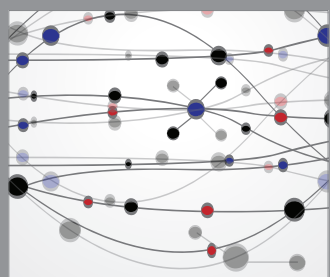

The Scientific World Journal
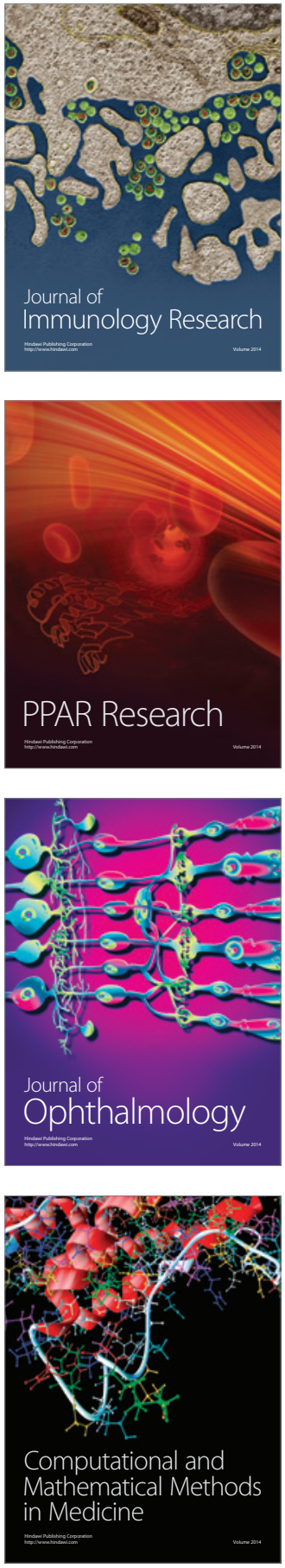

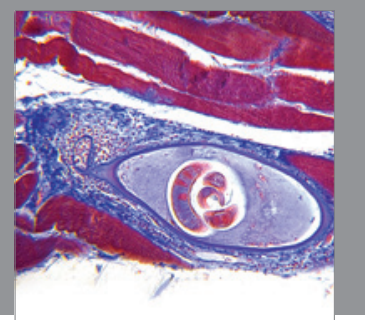

Gastroenterology

Research and Practice
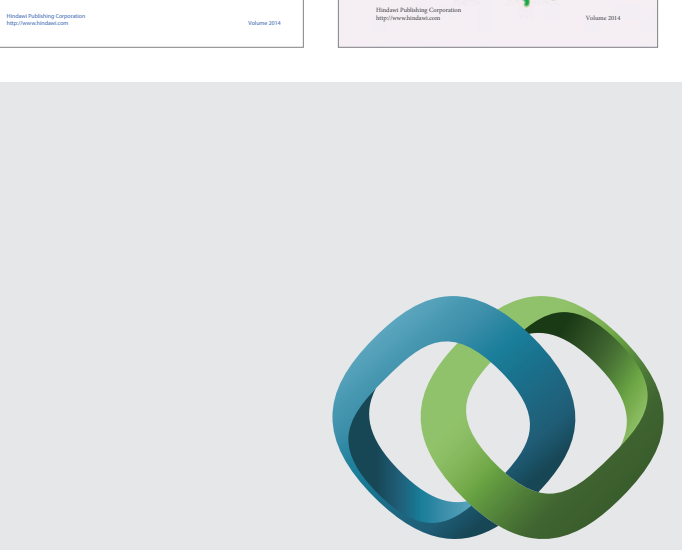

\section{Hindawi}

Submit your manuscripts at

http://www.hindawi.com
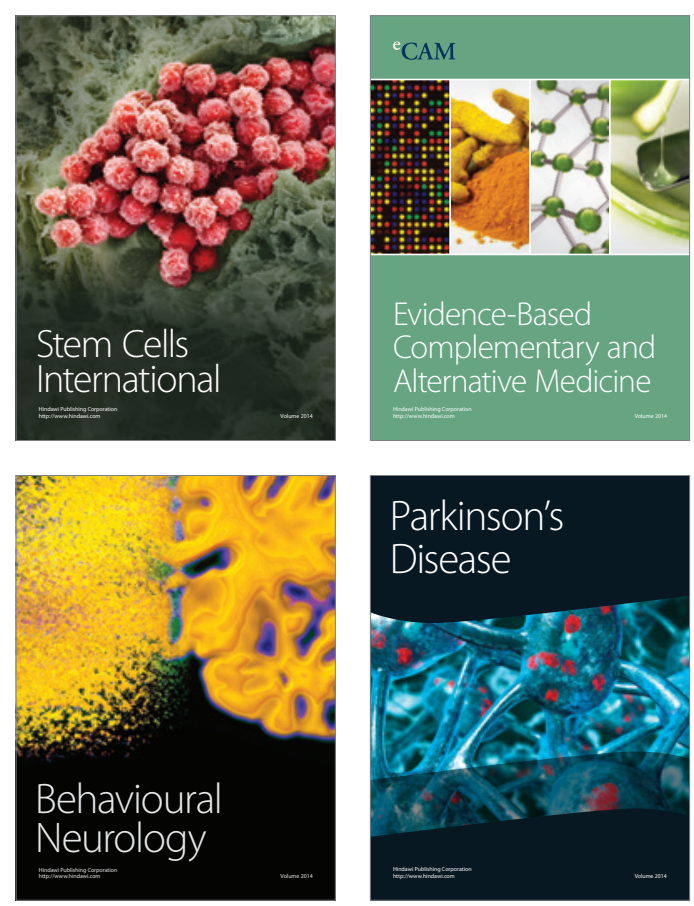

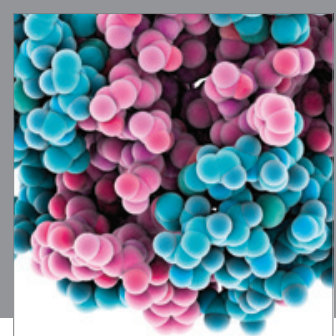

Journal of
Diabetes Research

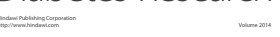

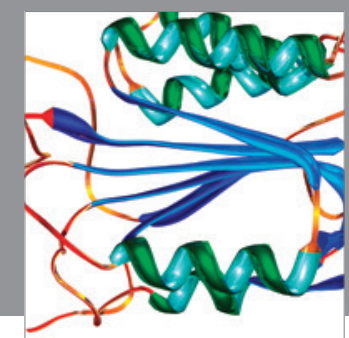

Disease Markers
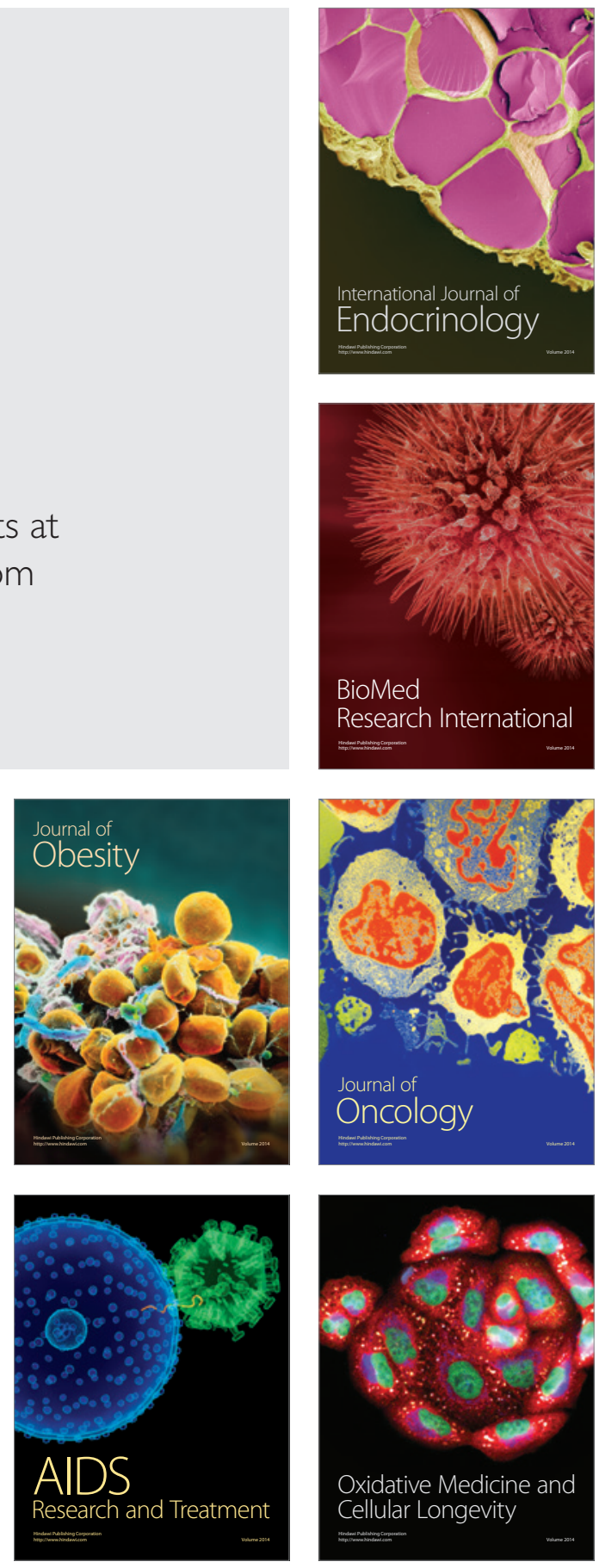